\title{
Temporal grouping and presentation rate in serial recall by retarded and nonretarded children
}

\author{
GILBERT J. HARRIS \\ Herbert H. Lehman College, City University of New York, Bronx, New York 10468 \\ and \\ DEBORAH BURKE \\ University of Chicago, Chicago, Illinois 60697
}

\begin{abstract}
Retarded and nonretarded subjects heard temporally grouped and ungrouped sequences of digits at varying presentation rates in a serial recall task. The results showed that the facilitatory effect of temporal grouping was not as great for retarded subjects as for nonretarded subjects younger in mental age. Several possible explanations for the smaller effect of temporal grouping for the retarded subjects were discussed.
\end{abstract}

Since Miller's (1956) influential paper, explanations of human short-term memory performance have centered on the subject's ability to organize the material to be remembered or to take advantage of any organization inherent in it. One area of research activity for those interested in the development of such organizational processes in children, and the possible lack of them in retarded subjects, is concerned with the experimental imposition of spatial or temporal organization on material to be serially recalled.

Several studies (MacMillan, 1970a, b; Spitz, 1966) comparing retarded and equal mental age nonretarded children have imposed spatial organization on visually presented digit sequences by pairing the digits during presentation. Results from these studies show no facilitation of recall with grouped input for nonretarded children but varying degrees of improvement for retarded subjects. It was argued that nonretarded subjects spontaneously group the ungrouped sequences, and thus obtain less benefit from the experimenter-imposed organization than do retarded subjects, who do not spontaneously organize the ungrouped material. Subject-initiated organization of the ungrouped sequences is reasonable in these studies given the relatively slow presentation rates of the material $(2.5-3.0 \mathrm{sec} / \mathrm{item})$. It has been argued that slower rates of presentation for visually presented naterial increase memory performance because there is more time for rehearsal and organization (e.g., Posner, 1963).

Requests for reprints should be sent to Dr. Gilbert J. Harris, Department of Psychology, Herbert H. Lehman College, City University of New York, Bedford Park Boulevard West, New York. New York 10468. This research was partially supported by Postdoctoral Fellowship MH-02581 from the National Institute of Mental Health. United States Public Health Service, to the second author.
Harris (1972) investigated the serial recall of six digits when visually presented simultaneously (ungrouped) or successively in two groups of three (temporally grouped). Total presentation time for both conditions was $9 \mathrm{sec}$, or $1.5 \mathrm{sec} /$ item. The results showed that nonretarded subjects, lower than the retarded subjects in mental age, had higher recall than the retarded subjects for the grouped but not for the ungrouped sequences. Harris and Burke (1972) compared second, fourth, and sixth grade children on serial recall of nine digits visually presented for $15 \mathrm{sec}$. Temporally grouped sequences, but not spatially grouped sequences, yielded higher recall than ungrouped sequences across all three age levels. These results and those of Harris (1972) suggest that temporal grouping of visually presented sequences is of more benefit to nonretarded children than is spatial grouping. Further, at least for adults, the facilitative effect of temporal grouping is even more marked using auditory rather than visual presentation (Talland, 1968). Indeed, the facilitatory effect of temporal grouping with auditory presentation does not seem to vary with age and has been found with children as young as 4 years (Huttenlocher \& Burke, 1976). One of the purposes of the present study was to determine whether such an effect may be obtained with retarded subjects.

Contrary to visually presented material, auditory material presented at fast rates produces higher recall than at slower rates (e.g. Conrad \& Hille, 1958; Mackworth, 1965). Mackworth (1965) has suggested that the advantage of fast presentation rates with spoken auditory sequences is due to the intonations and rhythms of normal speech which are difficult if not impossible to eliminate and which become more marked the faster the rate. She provided evidence for this hypothesis by showing 
that, when these intonations and rhythms are removed mechanically, the facilitative effect of fast presentation rates is reduced. Mackworth's finding, along with the result of Harris (1972), that the retarded are less able to benefit from temporal organization suggests that, in addition to profiting less from temporal grouping than do nonretarded subjects, retardates should show less increase in serial recall for fast presentation rates of ungrouped sequences than do equal mental age normal subjects. One study, however, has found that the facilitative effect of fast presentation rates for auditory material is the same for retarded subjects as it is for both their mental age and chronological age controls (Frankel \& Tymchuk, 1974).

The present study investigated the serial recall of temporally grouped and ungrouped auditory digits to determine whether such organization was more effective for normal than for retarded subjects, as was found by Harris (1972) using visually presented material. The effect of presentation rate, which for spoken material may be related to the organization inherent in normal speech, was also investigated. In addition. if earlier studies are correct in suggesting that the benefit of experimenter-imposed grouping is reduced at slower presentation rates, then grouping effects should be greater the faster the rate.

\section{METHOD}

\section{Design}

A 3 by 2 by 3 by 6 factorial experimental design was employed, with three groups of subjects (retarded adolescents, nonretarded 7-year-olds and 8-year-olds) constituting a between-subjects variable. The remaining three independent variables, input organization (grouped and ungrouped sequences), presentation rate $(1,2$, and 3 items/sec) and serial position within the six-digit sequences, were varied within subjects.

\section{Subjects}

The retarded subjects were 24 institutionalized male cultural familial adolescents $(C A=16.8$ years, $S D=2.7 ; M A=8.8$ years. $\mathrm{SD}=.8 ; \mathrm{IQ}=62.3, \mathrm{SD}=4.2)$. Nonretarded subjects were 24 male second graders $(C A=7.6$ years, $S D=.4)$ and 24 male third graders $(C A=8.6$ years, $S D=.3$ ) from an urban elementary school. The MAs for the retarded subjects were taken from the Stanford-Binet or estimated from the Wechsler Intelligence Scale for Children (WISC) or Adults (WAIS). For the two nonretarded samples, MA was assumed equal to CA.

\section{Materials}

The test materials consisted of 12 sequences, each made up of six nonrepeating digits trom the set 123456789 , with two grouped and two ungrouped sequences at each of the three rates. The sequences were tape recorded by the male experimenter, monitoring through headphones a metronome, set at 1,2 , or 3 beats/sec. For ungrouped sequences, one digit was recorded with the onset of each of six metronome beats, and a bell ring serving as the subject's signal to respond was recorded with the onset of the seventh. For grouped sequences, the first three digits were grouped such that the first digit was recorded with the onset of the first metronome beat and the third digit was recorded with the onset of the second. The second group of three digits began with the onset of the fifth beat, with the final digit coinciding with the onset of the sixth beat. The bell ring again was recorded with the onset of the seventh beat. The final input tape contained the 12 sequences in a random order, with the restriction that none of the six conditions (presentation rate by input organization) occurred twice before any one had occurred for the first time.

Subjects gave written responses on sheets containing six connected boxes ( $89 \mathrm{~mm}$ high $\mathrm{x} 38 \mathrm{~mm}$. wide) in a horizontal row.

\section{Procedure}

All the subjects were tested individually by a single female experimenter. Subjects were told they were going to play a "memory game" in which they would hear some numbers through headphones and would have to write them on the response sheet in the exact order in which they were presented. Subjects received two practice sequences, one grouped and one ungrouped, to assure their understanding of the procedure.

\section{RESULTS}

The dependent variable was the number of digits recalled correctly in the proper serial position. The mean number of items recalled correctly, collapsed across serial position, is shown in Figure 1, while the serial position curves collapsed across rate, are shown in Figure 2.

A 3 by 2 by 3 by 6 analysis of variance on the data showed a main effect of input organization $[F(1,57)=243.69, p<.001]$, indicating that grouped sequences were recalled more accurately than were ungrouped sequences. An interaction between the input organization variable and the groups factor $[F(2,57)=4.35, p<.01]$ resulted from the performance of the retarded subjects being superior to the 7 -year-clds for the ungrouped sequences but poorer for the grouped sequences. The main effect of presentation rate was significant $[\mathrm{F}(2,114)=37.99$, $\mathrm{p}<.001)$, with a subsequent Newman-Keuls analysis $(\alpha=.01)$ showing that the fastest rate produced the highest level of recall and the slowest rate the least recall. The triple interaction between input organization, groups, and presentation rate was also significant $[F(4,285)=3.46, p<.01)$, its locus being the finding that, for ungrouped sequences, the

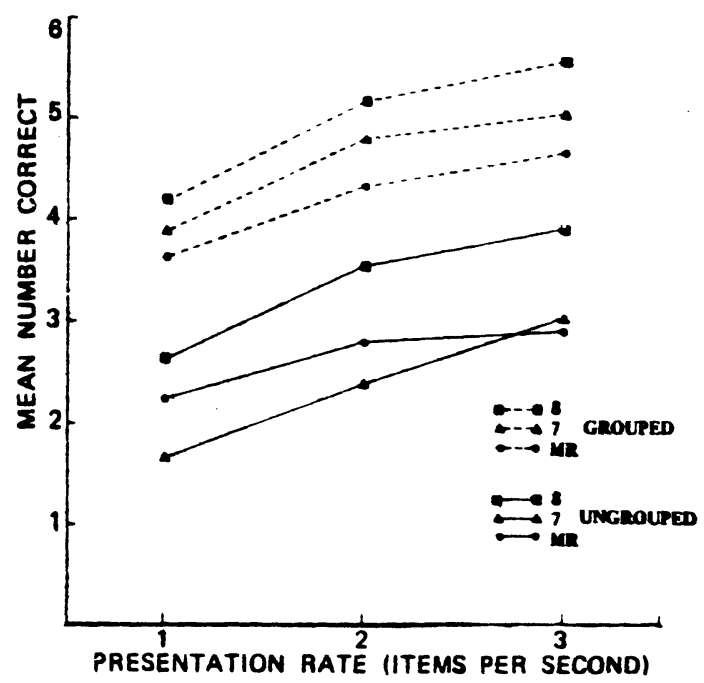

Figure 1. Mean number of items correct per sequence as a function of presentation rate, grouping, and groups of subjects. 


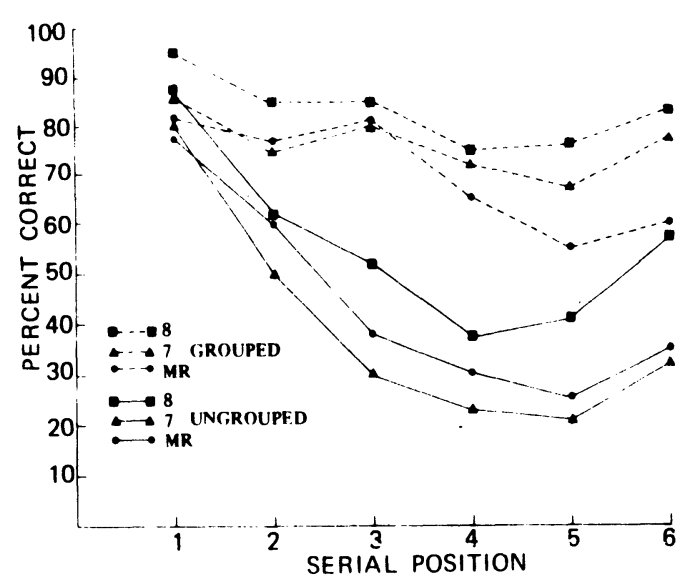

Figure 2. Percentage of items correct as a function of serial position, grouping, and groups of subjects.

performance of the retarded subjects fell below that of the 7-year-olds for only the fastest rate of presentation. It should be noted that there was no overall interaction between input organization and presentation rate, and it is clear from Figure 1 that the magnitude of the grouping effect was insensitive to rate.

The main effect of serial position was significant $[F(5,285)=78.93, p<.001]$, as indicated by the usual bow-shaped curves shown in Figure 2. A Groups by Input Organization by Serial Position interaction was obtained $[\mathrm{F}(10,285)=4.23$, $\mathrm{p}<.001]$, indicating that the locus of the Input Organization by Groups interaction occurred in the final three serial positions.

\section{DISCUSSION}

Temporal grouping greatly improved serial recall for all subjects at all presentation rates. In view of previous findings that spatial grouping with visual presentation had little or no effect on recall of retarded or nonretarded subjects, it seems that temporal grouping is a more salient form of imposed organization. However. the Groups by Input Organization interaction, obtained in the present study, supports the finding of Harris (1972) that grouping was less effective for retarded subjects than for nonretarded subjects. As Figure 1 shows, retarded subjects had generally higher performance than nonretarded 7-year-olds for ungrouped sequences, but this difference was reversed for the grouped sequences. The only deviance from this pattern of results was that the retarded subjects also had poorer performance than the 7-yearolds at the fastest presentation rate for the ungrouped sequences. This latter finding, which is contrary to the lack of interaction between groups and presentation rate found by Frankel and Tymchuk (1974), is consistent with the hypothesis that rapidly presented spoken sequences are easier to recall because they contain rhythmic temporal organization which can be useful to the listener. According to this explanation of the present results, the retarded subjects benefited less from the rapid presentation of the ungrouped sequences, and from the temporal grouping, because they have a poorer capability than do normals for taking advantage of organization inherent in presented material.

It is not obvious why the retarded are less able to benefit from temporal grouping and fast presentation rates. Indeed, there is no consensus as to the explanation of the facilitatory effect of temporal grouping on serial recall (e.g.. Bower \& Winzenz, 1969; Huttenlocher \& Burke, 1976). Possibly, retarded subjects possess a limited perceptual ability to abstract out structure in a sequence of items and are thus less able to make use of this structure in encoding item or order information. Alternatively, even though the organization in a sequence may be perceived and encoded, it may be lost in storage. When the retarded subject attempts to retrieve items, he might revert to an item by item strategy, losing some of the advantage the organization provides for nonretarded subjects.

A third possible explanation is suggested by the serial position curves. The present study showed that the retardates' recall deficit for grouped sequences, as compared with that of the 7-year-olds, was found only in the second group of three digits. Harris (1972) found that the deficit in the grouping condition for retardates was also localized in one group but, in that case, the first group.

It is probable that the part of the sequence that shows the greatest difference between retardates and normals in the grouped condition is determined by experimental parameters, such as presentation modality and presentation rate, which differed in the two studies. The finding in both studies, however, that retarded subjects did as well as nonretarded subjects on one of the two groups of digits, is consistent with the view that grouping structure may be as salient for retarded as for nonretarded subjects. It may simply be that retardates have a memory capacity limitation (Spitz, 1973) which restricts the overall effect of the imposed grouping.

\section{REFERENCES}

Bower, G., \& Winzenz, D. Group structure, coding and memory for digit series. Journal of Experimental Psychology Monograph, 1969, 80, No. 2, Part 2, 1-17.

Conrad, R., \& Hille, B. A. The decay theory of immediate memory and paced recall. Canadian Journal of Psychology, $1958,12,1-6$.

Frankel, F., \& Tymchuk, A. J. Digit recall of mentally retarded and nonretarded children under three presentation rates. American Journal of Mental Deficiency, 1974, 79. 311-319.

HARRIS, G. J. Input and output organization in short-term serial recall by retarded and nonretarded children. American Journal of Mental Deficiency, 1972, 76, 423-426.

HARRIS, G. J., \& BURKE, D. The effects of grouping on shortterm serial recall of digits by children: Developmental trends. Child Development, 1972, 43, 710-716.

Huttenlocher, J., \& Burke, D. Why does the memory span increase with age? Cognitive Psychology, 1976, 8, 1-31.

MACKWORTh, J. F. Presentation rate, repetition and organization in auditory short-term memory. Canadian Journal of Psychology, 1965, 19, 304-315.

MacMillan, D. L. Comparison of nonretarded and EMR children's use of input organization. American Journal of Mental Deficiency, 1970, 74, 762-764. (a)

MacMillan. D. L. Effect of input organization on recall of digits by EMR children. American Journal of Mental Deficiency, 1970, 74, 692-694. (b)

Miller, G. A. The magical number seven plus or minus two: Some limits on our capacity for processing information. Psychological Review, 1956, 63, 81-97.

PosNer, M. I. Immediate memory in sequential tasks. Psychological Bulletin, 1963, 60, 333-349.

SpITz, H. H. The role of input organization in the learning and memory of mental retardates. In N. R. Ellis (Ed.), International review of research in mental retardation (Vol. 2). New York: Academic Press, 1966.

SpITz. H. H. The channel capacity of educable mental retardates. In D. K. Routh (Ed.), The experimental psychology of mental retardation. Chicago: Aldine. 1973. Pp. 133-156.

Talland, G. A. Age and the span of immediate recall. In G. A. Talland (Ed.), Human aging and behavior. New York: Academic Press, 1968.

(Received for publication April 12, 1976.) 